\title{
APROXIMACIONES AL TURISMO ENOLÓGICO Y SUS ESTRATEGIAS DE MERCADOTECNIA EN MÉXICO
}

\author{
José Gabriel Ruiz Andrade \\ Universidad Autónoma de Baja California \\ gabruiz@uabc.mx \\ Omaira Cecilia Martínez Moreno \\ Universidad Autónoma de Baja California \\ omairam@uabc.mx \\ Ricardo Verján Quiñonez \\ Universidad Autónoma de Baja California \\ ricardoverjan@uabc.mx \\ Jorge Valderrama Martínez \\ Universidad Autónoma de Baja California \\ jorgevalderrama1@yahoo.com.mx
}

\section{RESUMEN}

El turismo es conceptualizado como un servicio que puede ser ofertado como un producto turístico compuesto por diferentes elementos complementarios; en este último caso habrán de agregarse atributos que complementen esta actividad con otras que se relacionen con la experiencia que va a vivir el turista o visitante, ya que éste se verá involucrado dentro de un ambiente donde interactuará con personas y con un ámbito geográfico, así como servicios adicionales que le permitirán obtener de esta combinación una vivencia que forme parte de sus vacaciones o tiempo dedicado a la recreación personal.

Existe actualmente un tipo de actividad turística especializada llamada turismo enológico, el cual es una forma de turismo que tiene varias connotaciones conceptuales; en cierto sentido pertenece al turismo rural, ya que es posible interactuar con comunidades que se encuentran dentro de la zona rural y en las que es posible realizar visitas a los viñedos, aprender los procesos de cultivo de la vid, su procesamiento para la elaboración del vino y su degustación.

La mercadotecnia aporta elementos que deberán considerarse al hacer una mezcla adecuada de las variables que inciden de manera directa en la formulación de estrategias para comercializar el turismo enológico.

Palabras clave: Turismo, enología, estrategias, mercadotecnia, mezcla 


\title{
AN APPROACH TO MARKETING STRATEGIES FOR WINE TOURISM IN MEXICO
}

\author{
José Gabriel Ruiz Andrade \\ Universidad Autónoma de Baja California \\ gabruiz@uabc.mx \\ Omaira Cecilia Martínez Moreno \\ Universidad Autónoma de Baja California \\ omairam@uabc.mx \\ Ricardo Verján Quiñonez \\ Universidad Autónoma de Baja California \\ ricardoverjan@uabc.mx \\ Jorge Valderrama Martínez \\ Universidad Autónoma de Baja California \\ jorgevalderrama1@yahoo.com.mx
}

\begin{abstract}
Tourism as an activity is defined as a service that can be offered as a tourist product comprised of different elements that improve such action with experiences that tourists or visitants may enjoy while surrounded by an environment where they can interact with community members, a geographical scenario and services that could be part of their leisure time or vacation.
\end{abstract}

Currently, there is a new tourist activity named wine tourism, which is a form of tourism that has many conceptual implications, but due to its characteristics it is related to rural tourism or alternative tourism, others are considering it as part of a new kind of classy tourism.

Marketing helps with tools that may be considered while mixing marketing for services; these variables may have direct impact on strategies when trading with wine tourism as a product.

Key words: Tourism, enology, strategies, marketing mix 


\section{INTRODUCCIÓN.}

La actividad turística conocida como enoturismo une dos elementos que dan origen a su nombre, de la parte de la enología tiene la relación con la elaboración técnica del vino, donde se incluye su procesamiento, almacenamiento, las formas en que se conserva, embotellado y comercialización; por otra parte, del turismo obtiene las ventajas de los servicios que pueden ser utilizados para complementar la experiencia en el conocimiento de los viñedos, las bodegas de añejamiento y las marcas de las diferentes compañías productoras; esto sucede al agregar los medios de transporte (terrestre y aéreo), guías especializadas, hospedaje (posadas, hoteles, casas de huéspedes, zonas de acampar) y alimentos y bebidas (restaurantes).

Dentro del complejo concepto del enoturismo, las estrategias de mercadotecnia que pueden ser utilizadas en la comercialización de los servicios y productos turísticos relacionados con esta actividad, tienen la oportunidad de generar elementos que permitan el conocimiento de la misma y que relaciona al turismo con la enología y las ventajas que un nuevo producto generan dentro del mercado turístico.

\section{ANTECEDENTES.}

Turismo es conceptualizado como un servicio que puede ser ofertado como producto turístico, el cual esté compuesto por diferentes elementos complementarios; en este caso habrán de agregarse atributos que enriquezcan este último con otros que se relacionen con la experiencia que va a vivir el turista o visitante, ya que éste se verá involucrado dentro de un ambiente donde interactuará con personas y con un ámbito geográfico, así como actividades adicionales que le permitirán obtener de esta combinación una vivencia que forme parte de sus vacaciones o tiempo dedicado a la recreación personal.

Existe actualmente un tipo de actividad turística especializada llamada turismo enológico, el cual es una forma de turismo que tiene varias connotaciones conceptuales, ya que pertenece al turismo rural, siendo posible interactuar con comunidades que se encuentran dentro de la zona rural y en las que se hace necesario realizar visitas a los viñedos, aprender los procesos de cultivo de la vid, su procesamiento para la elaboración del vino y su degustación. 
El turismo rural involucra a las personas de la comunidad con los turistas facilitando el contacto social en un medio donde se tiene la posibilidad de interactuar con la agricultura y conocer en este caso el proceso de elaboración de un producto agrícola por medio de relaciones cercanas y formativas con las comunidades agrícolas que los producen.

Por su parte, (Secretaría de Turismo de México, 2008) define al turismo alternativo como "los viajes que tienen como fin realizar actividades recreativas en contacto directo con la naturaleza y las expresiones culturales que le envuelven con una actitud y compromiso de conocer, respetar, disfrutar y participar en la conservación de los recursos naturales y culturales”, el concepto anterior envuelve al ecoturismo de manera que permite debido a sus características con la interacción del entorno natural a manejarse dentro de un aspecto más especializado y de acuerdo con los gustos e intereses de los turistas o visitantes. Así mismo, establece que éste agrupa tres grandes segmentos, cada uno compuesto por diversas acciones, donde cabe mencionar, que cualesquiera de las actividades que a continuación se definen, puede requerir de guías, técnicas y equipo especializado: ecoturismo, turismo de aventura y turismo rural.

El turismo rural está integrado dentro del turismo ecológico, el cual engloba las actividades turísticas que se llevan a cabo con el propósito de hacer turismo en zonas naturales, en donde la interpretación y observación de la naturaleza, así como su preservación son parte de los elementos que forman parte de esta alternativa. El enoturismo incluye elementos de turismo rural, dado que su entorno inmediato es este ámbito; pero al llevarse a cabo dentro de zonas que se pueden explotar de manera racional y con fines de preservación caen dentro del ecoturismo, este último clasificado dentro del concepto de turismo alternativo.

La Secretaría de Turismo de México no considera en su clasificación de turismo alternativo al enoturismo, sin embargo sí está incluido por el tipo de actividad que se realiza, diferenciándose por el enfoque a la producción de vino, el conocimiento de los procesos de cultivo y de las áreas geográficas en las que se realiza (turismo rural), permitiendo a los turistas o visitantes un contacto con las zonas naturales circundantes a las zonas de cultivo (ecoturismo) donde se da esta actividad rural especializada; lo anterior se genera por medio de desplazamientos de personas cuyo propósito es disfrutar del entorno por medio de actividades relacionadas con la cata de vino, los recorridos guiados por las bodegas y por el conocimiento de las características que le dan valor agregado a cada uno 
de los productos que son conocidos en su origen. Esta experiencia que se ve enriquecida con diversos servicios turísticos permite una oportunidad para disfrutar con mayor detalle y durante más tiempo la visita a las zonas productoras de vino.

Una descripción del concepto de enoturismo en (Turismo de vino, 2009) dice que el turismo de vino es una forma de hacer de esta actividad una en la que el atractivo principal son las visitas a bodegas y viñedos, unidas al disfrute de ellos y de todas las comodidades que ofrece el sector turístico: restaurantes con buena comida, hoteles agradables, actividades culturales o al aire libre, festivales, etc.

Por su parte (Medina, 2008) define que el turismo del vino (o enoturismo) se distingue del turismo enogastronómico sobre la base del objetivo prioritario de la visita; en el primero, el vino y su cultura se encuentran en primer término en relación con los intereses del visitante. En relación con el segundo, el vino y su cultura forman parte de los intereses gastronómicos más amplios del turista, quien utiliza el vino como un elemento más o menos principal de sus intereses culinarios, que acostumbran a ser más amplios (platos y/o productos locales, restaurantes específicos, rutas, etc); además agrega que cualquier «enoturista» es consumidor de restauración y está también posiblemente interesado en la gastronomía local o regional. La diferencia, sin embargo, es tanto de objetivo prioritario (el vino) como de grado o intensidad (lo relacionado específicamente con el vino y su cultura antes que con los elementos diversos de la gastronomía en general).

\section{DISCUSIÓN}

Una de las nuevas tendencias de la mercadotecnia es la basada en las sensaciones como lo menciona (Marquet, 2007), el consumidor de vinos con denominación de origen siempre va a buscar una experiencia única en la compra del producto y, en consecuencia, todas las actividades de marketing encaminadas a hacer sentir, oler, catar o vivir nuestro vino tienen las máximas garantías de éxito y resultan ser muy rentables para la compañía, el mismo autor considera que la gestión de las sensaciones es básica para cualquier detallista o empresa productora de vinos con denominación de origen, puesto que forma parte de la esencia de un punto de venta (la atmósfera de compra).

En este sentido se deberán de enfatizar los atributos que puedan lograr que la 
prestación del servicio aunado a la visita del lugar generen estímulos sensoriales a los visitantes de los viñedos, las bodegas y las áreas y negocios cercanos.

Una tendencia que se puede consolidar es el uso de las redes sociales en Internet, en este sentido (Gonzalvo, 2009) sugiere conseguir que los vinos de una bodega sean catados y comentados por unos cuantos bloggers, foreros o miembros de alguna red social, es una pequeña inversión que a corto plazo da a un vino una posición privilegiada que provoca reacción positiva en el público y genera confianza y cercanía en el consumidor. Estos consumidores satisfechos (por el vino y la información encontrada) se convierten en nuevos prescriptores que pueden hacer mucha más labor que otras formas de publicidad mucho más costosas.

Con el propósito de identificar oportunidades de mercado se debe conocer las tendencias que pueden sugerir qué negocios relacionados con cierta actividad serían las que tendrían mayores oportunidades de éxito. En este sentido (Marín, 2007) citando a Gustavo León manifiesta que en México el vino de mesa ha tenido un crecimiento más rápido que el tequila en los últimos 5 años, sin embargo la bebida nacional sigue siendo la categoría más importante, además agrega la misma fuente que según estimaciones de la Casa Pedro Domecq, el tequila tiene un crecimiento del 1\% anual y el vino, de 9\%”.

Por su parte (Marín, 2007) citando a la agencia BERG Marketing \& Research en Hábitos de Consumo y Compra de Vinos de Mesa, el Internacional Wine and Spirit Record (IWSR, 2006) y el INEGI menciona que "el consumo de vino en México se ha duplicado en los últimos ocho años a 500 mililitros per cápita. Es la categoría con mayor crecimiento constante, con el 9\% en los últimos 5 años. (IWSR, 2007) sostiene que "en México se consume más el vino tinto: la distribución es 66\% de tinto contra 34\% de blanco".

La producción de vino en México tiene una capacidad limitada de producción, siendo una actividad agrícola que recién empieza a ganar reconocimiento e importancia, la Oficina Económica y Comercial de la Embajada de España en México (2006) considera que el vino mexicano adquiere visibilidad a finales de los años 80 , y aunque su volumen de producción aún es reducido, se ha pasado de producir 10.476.000 L de vino de mesa en 1996 a 14.4000 .000 L.”; cita la misma fuente que "esta producción se reparte un 80\% en vino tinto, un $15 \%$ en vino blanco y un $5 \%$ en espumoso" y además agrega que "en 
su conjunto, las casas productoras elaboran más de 180 diferentes tipos de vino, entre tintos, blancos, rosados y espumosos. Estos vinos, se obtienen de 40 variedades de uva cultivadas en México, principalmente en las siguientes zonas vitícolas registradas: Baja California, Coahuila, Querétaro, Zacatecas y Aguascalientes”.

El potencial de consumo es grande, ya que es muy bajo a nivel nacional, (Gastronomía y Cía., 2009) dice que actualmente México ocupa el puesto número 65 en el consumo mundial de vino, donde además agrega que el consumo de esta bebida en el país es emergente como lo es en China o India, habiendo iniciado el desarrollo de la cultura del vino en el país, aumentando con ello las exportaciones. Como dato significativo cabe destacar que el $60 \%$ de todo el vino que se consume en México es importado, aunque este dato podría cambiar en los próximos años, ya que la producción de vino en el país crece en forma espectacular.

Con respecto a la posibilidad de desarrollar la actividad turística en condiciones favorables dentro de las zonas rurales (Verján, Valderrama y Ruiz, 2008:185) consideran que el desarrollo turístico sostenible debe significar para las estructuras locales el alcance de beneficios que puedan evidenciar el mejoramiento de las condiciones de vida de la población residente, además agregan que ofertar un producto turístico de calidad debe garantizar también el bienestar de los externos a la comunidad y por ende su valoración y acceso responsable, bajo criterios de sana y respetuosa convivencia con las comunidades receptoras y finalmente establecen que el manejo de sitios, áreas y zona turísticas bajo criterios de sostenibilidad se perfila como una posibilidad verdadera de mejora y crecimiento social.

En este sentido, no solo deberá de beneficiarse a las casas productoras de vino, sino que buscando el bien generalizado para las comunidades que estén integradas a las zonas vitivinícolas se vuelve conveniente el trabajo conjunto, donde la idea de conservación y preservación permita la utilización razonable de los atractivos de esas regiones.

\section{LA MEZCLA DE MERCADOTECNIA PARA EL ENOTURISMO}

Dentro de la mezcla de mercadotecnia es necesario definir el concepto de precio el cual describen (Stanton, Etzel y Walter, 2007:361) En cada empresa, el precio es 
un factor significativo para lograr el éxito en el marketing; y en muchas situaciones de compra puede ser de gran importancia para los consumidores. Sin embargo, es difícil definir el precio. Una definición general podría ser: el precio es la cantidad de dinero u otros elementos de utilidad necesarios para adquirir un producto.

El enoturismo es una actividad que se realiza al integrar una serie de elementos que van agregando un valor al precio final que se paga como un precio y que dependerá en cada caso de las partes que vayan a formar parte de la composición del mismo. Se incluyen costos de traslado, alimentación, hospedaje, servicio de guías, admisión a cavas o bodegas y otros servicios complementarios que pueden variar dependiendo de la oferta del lugar.

El precio puede determinarse como un paquete en el que se incluyan todos los servicios o pagarse de manera separada. Pueden ser ofertados desde las mismas bodegas vitivinícolas o por medio de intermediarios, ya sean agentes de viajes, tour operadores o profesionistas independientes o por medios electrónicos a través de páginas web o buscadores.

En este caso la experiencia de la visita a un viñedo y sus bodegas se complementa con la degustación y la eventual compra de vinos, esta última acción puede considerarse como parte del precio final por el uso de todos los servicios o como independiente, ya que la compra de un producto adicional supone una actividad no considerada dentro del paquete pero que constituye parte de la experiencia de la visita.

El precio puede verse influido por las marcas de los productos que se producen a partir de las casa vinícolas, donde el valor de la marca y de sus precios al público de sus productos puede diferenciar a un mercado, el cual espera pagar un precio de acuerdo con el valor de la marca de la compañía productora, lo anterior con base al valor percibido y las experiencias previas de contacto con los productos que de esa marca ya conoce o de las que al menos tiene referencia previa.

El valor que asigna un consumidor al precio estará determinado por el valor percibido que este tenga en relación con lo que recibe, como mencionan (Zeithaml, Bitner, Gremler 2009:524) una de las formas más apropiadas en que las compañías asignan un precio a sus servicios es basando éste en el valor del servicio que los clientes perciben. 
Dentro de este mismo contexto se determinan cuatro definiciones del cliente sobre el valor (Ver Fig. 1). Así mismo, estos autores consideran que para ciertos compradores o consumidores el precio bajo es una característica de valor en los productos o servicios, por lo que su intención de compra estará influenciada por precios bajos o que ofrezcan un descuento; para aquellos que piensan que valor es lo que desean de un producto o servicio, entonces la atención en la adquisición o consumo estará basada en los beneficios que obtienen a cambio, y por lo tanto para este caso la calidad debe de manifestarse de manera preponderante; con respecto a los que creen que valor es la calidad que obtienen por el precio que pagan tiene más que ver con el intercambio entre la cantidad pagada en dinero y los atributos que reciben, respondiendo en su caso a paquetes o productos que incluyen varios artículos o servicios integrados; y finalmente para los compradores que aprecian que valor es lo que obtienen a cambio de lo que dan, éstos incluyen el valor que le han asignado a todos los elementos que intervienen como costos no monetarios (tiempo, dinero y esfuerzo).

Figura $\mathrm{N}^{\circ}$. 1 Cuatro significados de valor percibido

\begin{tabular}{|c|}
\hline $\begin{array}{l}\text { Valor es precio } \\
\text { bajo }\end{array}$ \\
\hline \begin{tabular}{c} 
Valor es la \\
calidad que \\
obtengo por \\
precio que pago \\
\hline
\end{tabular}
\end{tabular}

\begin{tabular}{|c|}
\hline $\begin{array}{c}\text { Valor es todo lo } \\
\text { que obtengo a } \\
\text { cambio de todo } \\
\text { lo que doy }\end{array}$ \\
\hline
\end{tabular}

Valor es todo lo que deseo de un servicio

Fuente: Marketing de Servicios Zeithaml, Bitner, Gremler (2009).

Con respecto al producto (Kotler y Keller, 2006:372) consideran que son muchos los que piensan que un producto es una oferta tangible. Sin embargo, un producto es mucho más que eso. Un producto es todo aquello que se ofrece en el mercado para satisfacer un deseo o una necesidad. Los productos que se comercializan se dividen en productos físicos, servicios, experiencias, eventos, personas, lugares, propiedades, organizaciones, información e ideas.

El turismo enológico por si solo no es un producto, se integra al mezclar una serie de atributos que complementen una experiencia, la cual será más rica y duradera 
cuando se logre integrar elementos tangibles que hagan que la utilización de uno o varios servicios incorporen productos o situaciones memorables a través de las sensaciones para esa experiencia. Éste, incorpora los siguientes elementos: viñedos, visita a bodegas, servicio de degustación, alimentos, transporte, hospedaje, enseñanza de los procesos y sistemas para la elaboración de vino, eventos especiales (fiestas de la vendimia o festivales gastronómicos que incluyan vinos)

Los canales de mercadotecnia y las cadenas de valor se integran a la planta productiva como elementos que se integran para hacer llegar los productos o servicios a los clientes. (Kotler y Keller, 2007:468) sostienen que la mayoría de los fabricantes no vende sus productos directamente a los usuarios finales, sino que entre ellos existe una serie de intermediarios que realizan diversas funciones. Estos intermediarios conforman los canales de marketing (también llamados canales de distribución o canales comerciales). Formalmente, los canales de marketing son conjuntos de organizaciones interdependientes que participan en el proceso de poner a disposición de los consumidores un bien o un servicio para su uso o adquisición.

Los diferentes viñedos y bodegas productoras de vino tienen una características muy particular con respecto a la plaza, ya que si bien pueden tener representaciones o casas de venta en diferentes sitios, el viñedo y las bodegas serán encontradas en el sitio de producción, siendo éstas el principal atractivo del turismo enológico, por lo que la plaza se limitará a la zona geográfica en la que se produce el vino, teniendo la posibilidad de tener representaciones o canales de venta de los productos y servicios en diferentes lugares y por diversos medios de comunicación.

La integración de varios servicios y productos permiten contar con elementos atractivos para las diversas formas de publicidad que puede llegarse a hacer para el turismo enológico.

Las funciones de comunicación dentro de la promoción de productos o servicios según (Kotler y Keller, 2007:536) son el medio por el cual una empresa intenta informar, convencer y recordar, directa o indirectamente, sus productos y marcas al gran público. En cierto modo se podría decir que las comunicaciones de marketing representan la "voz" de la marca, y posibilitan el diálogo y la creación de relaciones con los consumidores”. Los mismos autores sostienen que "las comunicaciones de marketing desempeñan numerosas 
funciones para los consumidores, quienes, a partir de ellas, reciben información de la empresa sobre cómo y por qué se emplea un producto, quiénes lo utilizan, dónde y cuándo. Además, también se enteran de quién fabrica el producto y qué significan la marca y la empresa, y en último término, reciben un incentivo o una recompensa por probar o utilizar el producto. Las comunicaciones de marketing permiten a las empresas vincular sus marcas a personas, lugares, marcas, experiencias, sentimientos y objetivos, en este caso, las primeras contribuyen a recordar y transmitir la imagen de marca.

Los vinos, dentro de sus elementos de comunicación con el consumidor presentan ciertas características que los componen como productos procesados, los cuales se integran como insumo principal, siendo estos los atributos que los van a diferenciar de otras marcas y tipos de vino. Estos a su vez, se mencionan en la etiqueta dentro de los que se destaca: el origen del viñedo productor, la marca, el tipo de uva utilizada, el año de la cosecha, si ha sido acreedor a premios o distinciones especiales y la dirección de la casa productora, así como pueden incluir información para contactarles en caso de requerir más información, si es que se interesan en visitar determinadas regiones vitivinícolas, todos estos son parte importante de la estrategia de comunicación para fortalecer la marca y sus productos.

Las casas productoras de vino cuentan con páginas de Internet en las que se ofrece información más específica y en las que se pueden contratar servicios complementarios a la visita a los viñedos.

En regiones donde el turismo enológico se ha desarrollado de manera consistente como en España, Italia, Chile y Estados Unidos, se cuenta con agencias turísticas especializadas en la organización de paquetes promocionales, circuitos y recorridos guiados, además de páginas Web que funcionan como intermediarias en reservaciones y venta de estos productos turísticos.

En la mercadotecnia de servicios se agregan como variables que deben ser controladas por la empresa al personal de contacto, las instalaciones físicas y los procesos, esto con el fin de poder contar con elementos que controlados por la organización permitan ofertar un producto más rico en experiencias y con elementos tangibles que den un mayor valor agregado al turismo enológico. 
Toda organización debe de buscar que el personal de contacto esté capacitado e identificado con la empresa que representa, ya que se vuelve la parte visible de la misma, por lo que es una evidencia tangible de la prestación del servicio que está presente al contestar llamadas telefónicas, en mostradores o cajas registradoras, como guías o representantes de ventas, incluso en los viñedos pueden ser las personas que cultivan la tierra o tienen cuidado de la producción; si el personal va a tener contacto con los visitantes o turistas deben de contar con elementos que los distingan como parte de la empresa y que sean parte importante de la experiencia.

Elementos como el uniforme debe ser cuidado y de diseño particular para el viñedo o casa productora, tendiendo elementos en común que los identifiquen de otras empresas y en el que aparezcan logos o características de la organización.

La cortesía, empatía y buenos modos deben ser elementos que bien trabajados desde la empresa forman a prestadores de servicios comprometidos con su trabajo y logran proyectar una imagen de profesionalismo que hacen que la experiencia del visitante a los viñedos o bodegas se convierta en la oportunidad de evaluar a la empresa como un buen lugar para trabajar y que puede influir en la fidelización del consumidor al saber que la compra de productos de esa marca genera bienestar (al menos aparente) en sus empleados. Empleados que están cómodos en su trabajo proyectan seguridad para quienes los visitan.

Las empresas promotoras de visitas, talleres o excursiones a viñedos, bodegas y cavas deberán de evaluar el contacto realizado con los clientes antes, durante y después de la interacción cliente-empresa con el propósito de mantener la calidad de atención personal y guiar los esfuerzos encaminados a prestar un servicio con uniformidad.

Existen varios puntos que pueden estar en contacto físico con los visitantes, los cuales incluyen las instalaciones de las oficinas de ventas, los viñedos y bodegas y los medios publicitarios tanto físicos como electrónicos, además en los lugares donde se ofertan servicios de hospedaje y alimentación, se incluyen como parte de la experiencia tangible en su conjunto.

Dentro de esta categoría están los materiales impresos corporativos en los que se incluyen membretes, tarjetas de presentación, folletos informativos y cartas, estos materiales también pueden ser digitales para ser enviados por medio electrónico, 
siendo este medio uno de los más utilizados para realizar promociones y publicidad de la empresas productoras de vinos.

Los medios electrónicos permiten visualizar la empresa de manera virtual, de manera que sean las imágenes y la información las que inviten a disfrutar de la experiencia de catar un vino en el lugar que se produce o conocer el proceso de producción de una manera interactiva, mientras que otros pueden disfrutar del conocimiento profundo sobre las particularidades de una región vitivinícola en particular al conocer de forma personal y en sitio de un lugar de su interés.

Con respecto a la evidencia física (Zeithaml, Bitner, Gremler, 2009:313) consideran que debido a que los servicios son intangibles, los clientes con frecuencia confían en señales tangibles o en evidencias físicas para evaluar el servicio antes de comprar y evaluar su satisfacción durante y después del consumo, las mismas autoras mencionan que se incluyen todos los elementos tangibles de la instalación física de la organización (el ambiente del servicio) así como otras formas de comunicación tangible. Los elementos del ambiente del servicio que afectan a los clientes incluyen tanto atributos físicos exteriores (como señalización, estacionamiento y paisaje) y los elementos interiores (diseño, distribución, equipo y decoración)”.

El turismo enológico tiene la ventaja de ofrecer evidencias físicas al prestar sus servicios dentro de instalaciones que están relacionadas con sus productos tangibles y que forman parte de la experiencia dentro del proceso de degustación de vinos, visitas guiadas por personal de contacto, y materiales impresos, lo anterior se da dentro de ambientes geográficos agradables a la vista y con alto valor paisajístico.

Con respecto a las nuevas tendencias en el uso de la tecnología (Zeithaml, Bitner, Gremler, 2009:313) manifiestan que las páginas web y los ambientes de servicio virtuales a través de Internet son las formas más recientes de evidencia física que las compañías pueden utilizar para comunicar la experiencia del servicio al hacer esto más tangibles para los clientes antes y después de la compra.

En la mercadotecnia de servicios se debe de considerar al proceso o procesos que tiene que ver con la forma en que el cliente interactúa con la empresa, de forma tal que se pueda tener control sobre el mismo, (Zeithaml, Bitner, Gremler, 2009:25) conceptualizan 
al proceso como los procedimientos, los mecanismos y el flujo de actividades reales por los que el servicio es entregado: la entrega del servicio y los sistemas operativos.

\section{PROPUESTA DE ESTRATEGIAS PARA EL ENOTURISMO}

Si bien es cierto que en México se adopta el gusto por el vino a partir de la presencia española durante la época colonial, este no ha sido tan popular como el gusto por la cerveza o el tequila; existe gusto por los buenos vinos y hay un conocimiento tácito de la calidad con la cual se producen, pero hace falta incentivar su consumo. En este sentido, las empresas vitivinícolas conjuntamente con la Secretaría de Turismo, diseñan y desarrollan diversas actividades que promuevan el mismo; por ejemplo, cada vez son más populares las tradicionales fiestas de la vendimia, actividad que cada año se realiza en la época en que se cosecha la vid, las visitas a bodegas y casas productoras de vinos y en general, los eventos gastronómicos que traen asociado el consumo de vinos, tendiendo a incrementarse el mismo.

La cultura del vino encuentra una de sus mayores estrategias en el enoturismo, ya que se han unido dos actividades económicas importantes pero no muy asociadas entre sí: turismo y agricultura, específicamente turismo y enología, por lo que surge un nuevo producto turístico que fortalece fuertemente el consumo del vino de forma directa.

Una de las ventajas que tiene el vino es que tiene una connotación más saludable, particularmente los tintos, que son conocidos como antioxidantes, incluso se les ha llegado a considerar un medicamento, hay quienes los recetan de forma terapéutica y los recomiendan consumir frecuentemente con moderación. Deberán hacerse notar las bondades que genera para la salud el consumo responsable de estas bebidas, abrir el mercado hacia nuevos segmentos más informados y educados con respecto a las variedades, tipos de vinos y maridajes adecuados.

El producto como tal es conocido como vino, sus atributos como producto básico son color, aroma, textura, sabor; la botella como parte de producto ampliado genera una imagen de modernidad y buen gusto existiendo diferentes presentaciones tanto en botellas de cristal de diversos tonos, hasta tetra pack; la etiqueta informa sobre la cosecha, el origen y el nombre; los elementos anteriores carecen de significado si no se tiene la formación básica sobre el procesamiento del vino, si se desconocen los diferentes tipos de uvas y los 
sabores que pueden generarse partir del proceso de añejamiento y su origen, siendo en esta parte donde se logra un "plus" en el producto, es decir, se lleva al consumidor a ser un conocedor del producto por medio de la interacción con el proceso de producción, esta actividad se puede llevar a cabo por medio del uso de las tecnologías de la información o de manera presencial.

Es sin duda, la experiencia presencial la que lograría integrar más elementos al conocimiento del consumidor. Si bien es adecuado informarse por medios electrónicos, esto no es suficiente, ya que no permite ni la cata, ni la vivencia en situ a los viñedos. Esta ultima, crea confianza y puede generar fidelidad a la misma, además permite que la experiencia de la visita sea comentada de boca en boca y se genere a partir de esta actividad un mayor interés por el conocimiento de los productos y su eventual consumo.

El consumo responsable de bebidas alcohólicas es una prioridad en la sociedad actual, promoverlo es parte de la responsabilidad social de la empresa productora, ya que el propósito es formar consumidores frecuentes sin llegar a generar un vicio o dependencia al vino, sino mas bien lograr un consumo que aunque sea repetitivo, brinde beneficios a la salud, propicie una experiencia satisfactoria, e implícitamente el conocimiento de las propiedades y características de los vinos que consume.

Toda campaña promocional deberá mencionar las ventajas del vino y sus cualidades, pero invitando a los consumidores potenciales a hacer uso de su libertad para consumir vino de forma tal que no dañe su salud o el bienestar de otros.

\section{PRIMERAS REFLEXIONES}

El turismo enológico como actividad económica beneficia a las comunidades que cuentan con las condiciones ideales para su desarrollo ya que la conjugación de servicios turísticos facilita la experiencia de la visita a viñedos, bodegas y casas productoras de vinos, donde la cata forma parte del conocimiento que los clientes potenciales requieren para poder diferenciar las características de los productos que consume, en este caso vinos, y se logre a través de ese conocimiento una fidelidad a la marca, lo cual traería como consecuencia un incremento en la demanda y un mayor disfrute por parte de quien lo consume. 
En México, el turismo enológico se encuentra en la fase inicial, las casas productoras han logrado el reconocimiento nacional e internacional debido a la calidad de sus productos y han empezado a estimular el gusto por las experiencias enológicas, permitiendo el acceso a sus viñedos y bodegas, particularmente en Baja California, donde el gobierno del estado ha impulsado la llamada Ruta del Vino. La oportunidad para desarrollar el enoturismo en México es una buena opción, ya que se podrían generar las primeras aproximaciones para los interesados en aprender sobre la enología, estimular el consumo y formar a futuros consumidores responsables.

Una alternativa para controlar los momentos en que los clientes están en contacto con las evidencias físicas es por medio de la configuración de un esquema que muestre el flujo de actividades que pueden realizarse de manera ordenada, donde se pueda evidenciar los posibles contactos en cada paso de la prestación del servicio. De esta forma el personal sabe en todo momento en que parte del proceso se encuentra y puede monitorear a los clientes con el fin de prestar un servicio lleno de experiencias que sean notadas por los participantes dentro del proceso.

El turismo enológico puede verse mejorado por tres tipos de procesos que eventualmente mejoran la percepción de la experiencia como tal:

\section{Proceso de venta del destino}

Incluye los esfuerzos promocionales y de publicidad, donde el personal de contacto deberá de contar con elementos que les permitan atender con responsabilidad y proyectando seguridad en el conocimiento de los productos turísticos y servicios que vendan, ya sea como asesoría telefónica o en línea por medio de Internet.

Este proceso deberá de incluir fraseología ordenada para lograr uniformidad en la atención, tono adecuado de la voz y conocimientos sobre destinos de enoturismo y enología básica, esto con el fin de tener la información suficiente para interactuar de manera natural con los clientes.

Con respecto a la publicidad es necesario contar con una imagen congruente en los productos y servicios que se oferten, los mensajes deben comunicar con exactitud los atributos de las zonas en las que se realizará la visita de turismo enológico, así como una 
descripción de las características físicas y geográficas de la misma.

Determinar las estrategias de comunicación ayuda a tener control sobre el proceso de ventas, ya que guía a los consumidores a realizar una compra razonada o tener una decisión de visita a un destino enológico determinado.

\section{Proceso didáctico de elaboración del vino}

El proceso de elaboración del vino es considerado como información confidencial y de reserva para la empresa y los enólogos que colaboran en la elaboración del vino, pero para términos de este trabajo, se enfocará en el proceso que se muestra en cada una de las cavas y bodegas con respecto a la elaboración del vino y que sirve como estrategia didáctica para enseñar a los visitantes o turistas la forma en que se elaboran los diferentes tipos de vino y las mezclas y combinaciones que se realizan con el objetivo de obtener diferentes sabores, colores, olores, etc.

El proceso didáctico de la elaboración del vino incluye el saludo y presentación de las personas responsables de dirigir el recorrido por los diferentes momentos que requiere la producción del vino, esta etapa permite hacer notar las medidas de calidad que se llevan a cabo, con el fin de agregar valor a la experiencia en sí, pero apoyando a la marca y el prestigio de la empresa; la cata es parte central de la experiencia y puede llevarse a cabo siguiendo un protocolo específico y según hayas sido las características contratadas; en la parte del cierre pueden realizarse otras actividades complementarias y que sirvan como cierre de la actividad; puede estimularse la compra de vinos exclusivos o de reserva limitada.

\section{Proceso de la experiencia en el viñedo}

Si bien en el proceso didáctico se incluye una parte de la experiencia de el viñedo, esta es más compleja e involucra la experiencia previa a la llegada al sitio por visitar, la contratación de hospedaje en un lugar cercano, el tiempo de interacción en la cava, bodega y otras áreas de lugar, así como la contratación de servicios que hagan más larga la estancia en la zona.

Dentro de las actividades adicionales pueden encontrarse los servicios de alimentos y bebidas, paseos recreativos a pie o caballo, vinoterapia, relajación, talleres de gastronomía y enología, etc. 
La utilización de estrategias de mercadotecnia asociadas al enoturismo puede potenciar esta nueva actividad, siempre y cuando se logre mantener el control sobre las variables que forman parte de la mezcla de mercadotecnia de productos y servicios, de tal forma que la experiencias obtenidas por el consumidor antes de la compra de los vinos, su consumo y la posibilidad de extender su conocimiento a través de las visitas a los sitios productores en sus escenarios reales permitan el disfrute de una actividad relacionada con el conocimiento y profundización de la viticultura a través de la interacción con los elementos que componen este producto turístico y que genere posibilidades de negocio en los que se pueda involucrar a las comunidades cercanas a los viñedos y se diversifique la oferta de actividades complementarias conservando la sustentabilidad de estos destinos turísticos.

\section{REFERENCIA BIBLIOGRAFICA.}

Kotler, P. y Keller K. (2006). Dirección de marketing Duodécima edición. México: Editorial Pearson Prentice Hall.

Ruiz, A., J. Gabriel., et al (2008). Experiencias universitarias de acercamiento e involucramiento con las organizaciones. Primera edición. México: Editorial Ediciones ILCSA, S.A. de C.V.

Stanton, W., Etzel, M. y Walter, B. (2007) Fundamentos de marketing. Décimocuarta edición. México.: Editorial McGraw Hill.

Zeithaml, V., Bitner, M., Gremler, D. (2009). Marketing de servicios un enfoque de integración del cliente a la empresa. Quinta edición. México: Editorial McGraw Hill.

Secretaría De Turismo De México. (2008). Turismo alternativo una nueva forma de hacer turismo Segunda edición. México. SECTUR Fascículos de turismo alternativo, pp 7-54

\section{OTROS}

Instituto Nacional de Estadística, Geografía e Informática (2001). Banca electrónica. En: http://ciberhabitat.gob.mx/banco/elfuturo.htm

Gastronomía y Cia. (2009). El consumo de vino en México. En www.gastronomiaycia. com/2009/02/03/elconsumo-de-vinoe...

Ganzalvo, Juan Manuel (2009) Winebloggers y nuevas dinámicas prescriptitas. En: www.acenologia.com/cienciaytecnologia/winebloggers nueva_prescripcion_2cien0909.htm

Marín, Celia (2007) Se duplica consumo. En www.eluniversal.com.mx/nacion/151997. 
$\underline{\mathrm{html}}$

Marquet, Christophe. (2007) Las nuevas tendencias del mercado de vino en España. Facultad de Enología, Universidad Rovira i Virgili. En http://www.acenologia. com/ciencia87.htm

Medina, Xavier (2008) Cultura de vino, gastronomía y enoturismo. Institut Europeu de la Mediterránea (IEMed), Barcelona, España. En http://www.acenologia.com/ ciencia99 1.htm

Turismo de Vino. (2009) ¿Qué es el enoturismo? En el portal de enoturismo de España. En http://www.turismodevino.com/enoturismo.php

Recibido: 21/07/2011

Aprobado: 31/10/2011

Arbitrado anónimamente 